\title{
Cortical waves mediate the cellular response to electric fields
}

\author{
Authors: Qixin Yang ${ }^{1,2}$, Yuchuan Miao ${ }^{3}$, Leonard J. Campanello, ${ }^{1,2}$, Matt J. Hourwitz ${ }^{4}$, Abby L. \\ Bull $^{1,2}$, Peter N. Devreotes ${ }^{3}$, John T. Fourkas ${ }^{2,4}$, Wolfgang Losert ${ }^{1,2 *}$.
}

Affiliations:

${ }^{1}$ Department of Physics, University of Maryland, College Park, MD 20742

${ }^{2}$ Institute for Physical Science and Technology, University of Maryland, College Park, MD 20742

${ }^{3}$ Department of Cell Biology, Johns Hopkins University, Baltimore, MD 21218

${ }^{4}$ Department of Chemistry \& Biochemistry, University of Maryland, College Park, MD 20742

*Correspondence to: $\underline{\text { wlosert@ } \mathrm{umd} . e d u}$

\section{Abstract:}

The molecular mechanisms by which electric fields direct cell migration remain largely unknown, because the cortical waves of signal transduction and cytoskeletal activity that drive cell protrusions are comparable in size to the cells themselves. To address this problem, here, we employ electro-fused giant cells, which are considerably larger than these waves, and nanotopography, which decreases the lifetime of waves on the basal surface. We find that electric fields increase the wave area and lifetime in the region of the cell nearer the cathode, and bias the wave direction. We also notice that shorter-lived waves on nanotopography lead to a faster cell response to electrical-field reversal. Our findings indicate that the directions and durations of waves have a dominant effect on cellular response to electric fields.

\section{One Sentence Summary:}

25 Electrotaxis is mediated by biased cortical waves, explaining why electrotaxis response is significantly slower than chemotaxis. 


\section{Main Text}

Electrotaxis, which refers to the directed migration of cells under the guidance of an electric field, is important in wound healing, development, and regeneration (1-3). EFs have been shown to cause several key signaling molecules to be distributed asymmetrically across cells (3-5), setting up cell polarity. The one-order-of-magnitude range of EF strengths sensed by cells (5) is considerably smaller than the four-order-of-magnitude concentration sensitivity in chemotaxis. Furthermore, cells respond to EF guidance on a time scale of minutes, but respond to chemical guidance cues on a time scale of seconds $(6,7)$. These differences raise the possibility that traditional gradient sensing pathways do not serve as primary mediators of EF sensing of cells. In this study, we examine whether instead biochemical excitable networks centered on actin polymerization comprise a slow-acting mediator of the cellular response to EFs.

Actin polymerization, in conjunction with the key signaling molecules that regulate guided cell migration, self-organizes into microscale spatial regions that travel as waves across plasma membranes and that drive protrusions $(8,9)$. The wave system can be described as a coupled signal transduction excitable network - cytoskeletal excitable network (STEN-CEN) (10, 11). This network has the characteristics of an excitable system, including exhibiting an activation threshold for wave initiation and experiencing refractory periods. A model of this activator/inhibitor, reaction/diffusion system successfully recreates diverse wave patterns and corresponding protrusion profiles observed across multiple cell types $(11,12)$. For simplicity, we will refer to STEN-CEN waves as cortical waves.

To account for the directed migration of these waves and protrusions toward external cues, a bias of the excitable network (BEN) was added to the STEN-CEN model (13). When external cues vary by order of magnitude in strength, sensing such a wide range of input strengths can be achieved by a combination of local excitation and global inhibition (LEGI) that translates surface receptor activation into downstream signals. The LEGI-BEN model has successfully explained the complex kinetics of response to chemoattractant (14). Recent studies have shown that electrotaxis and chemotaxis share the downstream signal pathways that regulate cytoskeletal activities for persistent polarity and motility $(5,15)$. However, the receptors that sense chemical gradients are not required for electrotaxis $(5,16)$. Furthermore, the kind of adaptation that can be achieved by the LEGI signal transduction system is not necessary in electrotaxis, which typically is only effective in a narrow range of gradients.

These observations raise the question of whether cortical waves are able to act as mediators of EFs. One challenge in addressing this question is that in many of the cell types that show a strong response to EFs, the wave area is comparable to the size of a cell. Furthermore, waves are generated at the leading edge of the cell during directed migration (17), so that wave dynamics are tightly coupled with cell dynamics. For instance, when a cell responds to an EF reversal, waves typically remain at the cell front as the cell turns. It is not known whether the waves drive cells to turn or the cell polarity keeps the previous leading edge more active so that this edge responds first. 
To distinguish between these two responses, we produced electro-fused giant D. discoideum (18) with diameters up to ten times larger than that of an individual cell. Multiple simultaneous waves can be generated across the surface contact area of a giant cell (8). These waves also generate actin-filled macropinosomes on the dorsal membrane (19). The giant cells provide an excellent opportunity to study cortical wave dynamics in multiple cell regions simultaneously. We further use nanotopography to alter the spatial structures and characteristic timescales of the waves. Upon contact with nanotopography, cells produce quasi-1D wave patches in a process known as esotaxis (20). These waves persist for a shorter time than do waves on flat surfaces, and thus, in giant cells, only propagate in local regions. Nanotopography therefore allows us to distinguish between local and global mediation of the EF response.

\section{Results}

\section{Actin waves and cell migration can be studied independently in giant cells}

We imaged cells that simultaneously expressed both limE-RFP, to monitor filamentous actin (Factin), which is the representative of CEN activities, and $\mathrm{PH}_{\mathrm{Crac}}-\mathrm{GFP}$, to monitor phosphatidylinositol-3,4,5-trisphosphate (PIP3), an indicator of STEN activities. In single, differentiated cells, only one wave was generated at the leading edge (Fig. 1A and Movie 1). When the cell changed its direction of motion, the wave remained at the leading edge (Fig. 1A, $72 \mathrm{~s}-120 \mathrm{~s}$ ). In giant cells, multiple waves were initiated randomly, and propagated radially across the basal membranes (Fig. 1B and Movie 2). As shown in Fig. 1B, waves that collided did not cross (Fig. 1B, $150 \mathrm{~s}-200 \mathrm{~s}$ ). This behavior is suggestive of a refractory period following excitation, which is a hallmark of an excitable system. On nanoridges, the giant cells generated multiple, quasi-1D patches of F-actin and PIP3 (Fig. 1C and Movie 3). As was the case on flat surfaces, 1D patches occurred throughout the basal surfaces, and thus were independent of cell motion.

Waves were also generated on the dorsal planes. In contrast with basal waves, which propagated across the surface contact (Movie 2 and Movie 3), dorsal waves were associated with membrane deformations, and resembled macropinosomes (Movie 4). Based on 3D lattice lightsheet images (Fig. 1B and Movie 5), activation of PIP3 and F-actin was correlated in dorsal waves. However, the dorsal waves were generated mostly in cuplike structures at cell boundaries. In all the cases, PIP3 activity was coordinated with F-actin activity (Profiles in Fig. 1A, 1B, and 1C). Therefore, in the experiments described in the remainder of the paper, we only monitored F-actin activity.

\section{Electric fields (EFs) increase the area, duration, and speed of waves.}

The 1D waves generated on nanoridges via esotaxis enabled us to quantify the effects of a 20 $\mathrm{V} / \mathrm{cm}$ EF (all EFs used here are of this magnitude) on wave properties including area, duration, and speed. Fig. 2A shows snapshots of the dynamics of F-actin in a giant cell on parallel nanoridges with a $1.6 \mu \mathrm{m}$ spacing. In the absence of an EF, individual actin polymerization events were initiated in patches on the basal surfaces (Fig. 2A and Movie 6). Most patches propagated as a wave along a single ridge (Fig. 2A, blue inset), but some patches appeared to exhibit coordinated motion across several ridges (Fig. 2A, pink inset). 
An EF increased the overall level of actin polymerization by a factor of two to three (Fig. 2B and Movie 6). Actin patches were larger in the presence of an EF, and organized into larger groups preferentially located at the cell front (Fig. 2A, $20 \mathrm{~min}$, and $25 \mathrm{~min}$ ), leading to wider protrusions at cell fronts that drove directed cell migration (Fig. S1). To determine whether the groups comprised a single large wave growing across multiple ridges or multiple small patches nucleated in close proximity, we measured the dynamics of the patches using optical flow (21), focusing on the patch edges (Fig. 2C, left image. See Methods for more details). If both edges of a patch were moving in the same direction, the structure was classified as a single large wave. If the edges were not coordinated, the patch was considered to be composed of multiple individual structures.

Once the large actin structures were classified, their instantaneous dimensions parallel and perpendicular to the ridges were measured (Fig. 2C). Density scatter plots of both dimensions exhibit elliptical contours (Fig. 2D), suggesting that nanotopography constrains wave growth. With an EF parallel to the ridges, the waves broadened in both directions (Fig. 2D). The average increases in wave dimension parallel and perpendicular to the ridges were $20 \%$ and $13 \%$, respectively, and the average increase in wave area was $44 \%$. An increase in wave duration was also observed, with the minimum wave area correlated to the duration (Fig 2E, black circles). The wave area depends exponentially on the maximum wave duration (Fig. 2E, black solid lines), allowing us to extract a characteristic wave time scale via

$$
\text { Area }_{\text {min }}=C * e^{\frac{\text { Duration }}{T}}
$$

Here $C$ is a constant and $T$ is the characteristic time scale. $T$ is $48 \mathrm{~s}$ with no EF to $61 \mathrm{~s}$ with a 20 $\mathrm{V} / \mathrm{cm}$ EF. This observation is consistent with the EF drawing the system closer to the excitability threshold. An average increase of $9 \%$ in wave propagation speed was also observed in the presence of an EF (Fig. 2F).

\section{EFs guide the direction of actin waves.}

We next consider the directional guidance of actin waves by EFs on nanoridges (Fig. 3A and Movie 6) and on flat surfaces (Fig. 3B and Movie 7, see Methods for details). The EF was introduced at time $T_{1}$, and in the first $2 \mathrm{~min}$ had little effect on the actin dynamics on any surface. On nanoridges, actin waves continued to propagate preferentially along the ridges (Fig. 3A, $\left.T_{1}\right)$. On flat surfaces, waves propagated radially in groups, as seen from the broad distribution at $T_{1}$ in Fig. 3B. In the presence of an EF, the waves propagated preferentially towards the cathode within $\sim 15$ min (Figs. 3A, 3B, $T_{2}$ ). The perpendicular spread was significantly more limited on ridges (Fig. 3A, $T_{2}$ ). 
The direction of the EF was reversed after the cell had commenced steady directional migration, which took $\sim 20$ to $25 \mathrm{~min}$. Following the field reversal, waves on ridged surfaces reoriented toward the new cathode within $5 \mathrm{~min}$ (Fig. $3 \mathrm{~A}, T_{3}$ ). On flat surfaces, the wave propagation direction was perpendicular to both the previous and new EF directions at $\sim 7 \mathrm{~min}$ after the field reversal (Fig. 3B, $T_{3}$ ). Preferential propagation towards the new cathode occurred after $\sim 13 \mathrm{~min}$ (Fig. 3B, $T_{4}$ ). The difference in response time between nanoridges and flat surfaces may be related to the fact that waves last longer on flat surfaces than on nanoridges (Fig. 3C, 3D).

In Figs. 3E and 3F we plot the continuous temporal changes of the orientation distributions. On nanoridges we observed direct switching of preferred wave directions following EF reversal (Fig. $3 \mathrm{E}$ ), whereas on flat surfaces waves maintained a preferred direction that changed continuously in a U-turn behavior (Fig. 3F). In contrast, although single $D$. discoideum cells undergo U-turns in response to EF reversal (22), giant cells did not (Movie 7).

Wave turning may be related to differences in the patterns of wave expansion (Fig. 3G and 3H). On flat surfaces, waves were observed to start from a small patch (Fig. 3H, S1), and eventually broke into band-shaped waves (Fig. 3H, S4). During directed migration, the intermediate expansion of actin waves (Fig. 3H, S2, S3 in top row) was biased by EFs, ending up with bandshaped waves preferentially propagating towards the cathode (Fig. $3 \mathrm{G}$ and $3 \mathrm{H}, \mathrm{S} 4$ in top row). After EF reversal, waves expanded in all directions (Fig. 3G and 3H, S2, S3 in bottom row), such that optical-flow analysis captured turning behavior more frequently (Fig. 3F).

We also simultaneously imaged limE-RFP at the basal plane (near the surface contact) and at the dorsal plane (6 $\mu \mathrm{m}$ higher). Dorsal waves (Movie 8) are localized at cell fronts and rearrange to the new fronts following EF reversal (Fig. S2B). Different from directly switching preferential direction, dorsal waves gradually turned toward the new cathode (Fig. S2D), in a manner similar to that of basal waves on flat surfaces. Thus, two different response times to EF reversal exist within the same cell, with a faster response for basal waves guided by nanotopography and a slower response for the free dorsal waves. On flat surfaces the two responses are synchronized in time (Fig. S3).

\section{Subcellular spatial inhomogeneity of the response to EFs.}

Although waves in migrating neutrophils and $D$. discoideum cells localize predominantly at the leading edge $(5,23)$, waves are observed across the basal layer in giant $D$. discoideum cells. We analyzed the smaller, shorter-lived waves on nanoridges. Although the wave locations were distributed essentially uniformly throughout cells without an EF, more waves were generated at the cell fronts in EFs (Fig. 4A and Movie 6). In addition, the average area per wave was larger near the front of cells in EFs (Fig. 4B). We further validated that this spatial inhomogeneity was caused by the polarized intracellular environments induced by EFs, instead of the external electrical potential gradient (Fig. S4). 
We explored the response of this inhomogeneity to EF reversal by tracking each wave location relative to the cell centroids in the 12 min following EF reversal (Fig. 4C). New waves started to appear near the side facing the new cathode within 3 min (Fig. 4C, left region of $P_{2}$ ), whereas the complete inhibition of wave generation near the old cell front takes longer (Fig. 4C, right region of $\mathrm{P}_{5}$ ). This observation suggests that the initiation at a new cell front and the inhibition of waves at the old front are regulated by two distinct processes with different time scales.

Next we looked at the time it takes to switch propagation direction to EF reversal in different subcellular regions. The basal membrane was segmented into an "old front" region (facing the original cathode) and a "new front" region (facing the new cathode), as illustrated in Fig. 4D. The distributions of wave propagation directions show that waves in the new front region switched their preferential direction at $\sim 4$ min, whereas waves in the old front region changed their preferential direction on a time scale of $\sim 7 \mathrm{~min}$ (Figs. $4 \mathrm{E}, \mathrm{F}$ ). Our analysis further shows that larger waves in the old fronts are less sensitive to EF reversal (Fig. S5).

\section{Discussion}

We have demonstrated that EFs directly affect the locations and directions of cortical waves. The wave system can be described as a coupled signal transduction and cytoskeletal excitable network. The wave ranges, durations and speeds are determined by the local thresholds of activation, which in turn are regulated by the relative levels of activators and inhibitors $(11,12)$. We employed giant cells, in which basal cortical waves and dorsal cortical waves coexist, and the former of which are disentangled from cell motion (Fig. 1 C, B, D). We further used nanoridges to generate quasi-1D waves that are small, short-lived, and unable to turn (Fig. 1C).

Our quantification shows that guided waves become larger, faster, and more persistent in an EF (Fig. 2), indicating that the excitable system is closer to its threshold for activation. This effect may arise from enhanced positive feedback, reduced negative feedback, or both. We further find that wave nucleation is enhanced at the cell front and suppressed at the back (Fig. 4A, B). This subcellular inhomogeneity is consistent with a BEN framework $(14,24,25)$, which introduces an internal spatial gradient in the local threshold of wave initiation, akin to cell polarity.

Previous studies have suggested that the basal cortical waves in D. discoideum are insensitive to external chemotactic gradients, whereas "pseudopods" at other regions in the same cells can be guided (33). This conclusion is surprising, because the biochemical events traveling with the waves are the same as those occurring on pseudopods, and are normally triggered by chemoattractant. Also, similar cortical waves in human mammary epithelial cells can be effectively guided by epidermal growth factors (34). Additional input from greater contact on the basal surface of giant $D$. discoideum cells may outweigh the effect of applied chemical gradients. Whatever the mechanism, it is clear that cells integrate combinations of external chemical and mechanical stimuli. On the other hand, electrotaxis has an even smaller dynamic range than chemotaxis, yet our work shows that waves in giant cells can indeed be guided by EFs (Fig. 3). 
In response to an EF reversal, the global rearrangement of wave initiation occurs on a time scale of $\sim 10$ min (Fig. 4C), whereas new waves are guided to propagate towards the current cathode within 2-3 min (Fig. 4E). This difference in characteristic times indicates that at least two mechanisms with different time scales are involved in electrotaxis. The faster directional response of waves reflects the shorter lifetimes of waves on nanoridges (Fig. 3C), and may be regulated by local changes in specific lipids, ion fluxes, or local $\mathrm{pH}$ gradients (26-30), all of which have been shown to regulate cytoskeletal activity.

There are several potential mechanisms for the slower global spatial rearrangement of wave nucleation. This phenomenon may result from electrophoresis of charged membrane components, which has a characteristic time scale of 5 to $10 \mathrm{~min}(31,32)$. This timescale is longer than the wave duration (Fig. 3C), but is consistent with the timescale for the establishment of cell polarity. Features of both directional sensing and stable polarity in response to chemical signals have been described successfully by LEGI models (14), which can predict robust internal gradients. Although G-protein-coupled receptors (GPCRs), which are the regulator in the LEGI model for D. discoideum to sense chemo-attractant on second-timescales, are not essential for electrotaxis (5), our results suggest that the polarity is still integrated into the response of STEN-CEN to EFs.

Our results suggest that the electrotaxis is regulated by a mechanism that is slower than that for chemotaxis but that has a stronger influence on STEN-CEN waves. This picture is consistent with the hypothesis that the cellular response to EFs is mediated by cortical waves. Waves travel across cell membranes to coordinate the trailing edge with the front edge, and the cytoskeletal components in cortical waves are involved in developing the stable polarity. On the other hand, the duration and turning capacity of STEN-CEN waves directly impact the speed and characteristics of the cellular response to EFs (Fig, 3) on a timescale that is longer than that of surface-receptors-regulated chemotaxis.

On flat surfaces, a slow U-turn is observed following EF reversal, whereas on nanoridges faster switching is observed. Thus, the response of migrating cells to a changing guidance cue may be predicted from the characteristics of the waves driving the migration process. Indeed, the U-turn behaviors of neutrophils and differentiated, single $D$. discoideum cells in response to EF reversal $(17,22,35,36)$, which is usually ascribed to stable cell polarity, may instead reflect the persistence and $2 \mathrm{D}$ behavior of cortical waves in these environments.

Nanoridges allow us to shed further light on the multiscale character of the system, because cells include both short, 1D waves on the basal plane, and longer-lasting, 2D waves on the dorsal plane. The coexistence of waves with different behaviors in different portions of a cell provides powerful evidence that cortical waves act as direct mediators of EFs. Waves on different planes are similar in composition, but they are impacted differently by the EF, with a fast switching of wave directions in the basal plane, and a slower turning of the waves in the dorsal plane within in the same cell indicating that the direction of waves is controlled locally by external cues (Fig. $\mathrm{S} 2)$. 
Taken together, EFs provide a means to modulate cortical waves directly. On the other hand, biological conditions that modulate wave characteristics may also speed up or suppress the cellular response to directional cues. Longer lasting waves offer persistence in the face of rapidly changing gradients, whereas shorter waves yield faster adaptability to changing directional signals. The durations of waves and their ability to turn together have a dominant effect on the response of cells to an EF.

\section{References and Notes:}

1. B. Cortese, I. E. Palamà, S. D’ Amone, G. Gigli, Integr. Biol. (United Kingdom). 6, 817830 (2014).

2. F. Lin et al., J. Immunol. 181, 2465-2471 (2008).

15 3. M. Zhao et al., Nature. 442, 457-460 (2006).

4. M. J. Sato et al., Proc. Natl. Acad. Sci. U. S. A. 106, 6667-6672 (2009).

5. M. Zhao, T. Jin, C. D. McCaig, J. V Forrester, P. N. Devreotes, J. Cell Biol. 157, 921-7 (2002).

6. M. Zhao et al., Nature. 442, 457-460 (2006).

20 7. M. J. Wang, Y. Artemenko, W. J. Cai, P. A. Iglesias, P. N. Devreotes, Cell Rep. 9, 11101121 (2014).

8. M. Gerhardt et al., J. Cell Sci. 127, 4507-4517 (2014).

9. S. Bhattacharya, Y. Miao, P. N. Devreotes, P. A. Iglesias, Biophys. J. 116, 121a (2019).

10. P. N. Devreotes et al., Annu. Rev. Cell Dev. Biol., in press, doi:10.1146/annurev-cellbio-

11. Y. Miao et al., Mol. Syst. Biol. 15, 1-20 (2019).

12. Y. Miao et al., Nat. Cell Biol. 19, 329-340 (2017).

13. P. A. Iglesias, P. N. Devreotes, Curr. Opin. Cell Biol. 24, 245-253 (2012).

14. M. Tang et al., Nat. Commun. 5, 1-13 (2014).

15. R. Gao et al., Sci. Signal. 8, 1-10 (2015).

16. J. Jiang et al., Sci. Signal. 8, 1-10 (2015).

17. J. Xu et al., Cell. 114, 201-214 (2003).

18. G. Gerisch et al., J. Cell Sci. 126, 2069-78 (2013).

19. U. Hacker, R. Albrecht, M. Maniak, J. Cell Sci. 110, 105-112 (1997).

20. M. K. Driscoll, X. Sun, C. Guven, J. T. Fourkas, W. Losert, 3546-3555 (2014).

21. R. M. Lee et al., Mol. Biol. Cell. 31, 1753-1764 (2020).

22. M. J. Sato et al., BioSystems. 88, 261-272 (2007).

23. O. D. Weiner, W. A. Marganski, L. F. Wu, S. J. Altschuler, M. W. Kirschner, PLoS Biol. 5, 2053-2063 (2007).

40 24. H. Meinhardt, J. Cell Sci. 112, 2867-2874 (1999).

25. Y. Xiong, C. H. Huang, P. A. Iglesias, P. N. Devreotes, Proc. Natl. Acad. Sci. U. S. A. 107, 17079-17086 (2010).

26. A. H. Crevenna et al., J. Biol. Chem. 288, 12102-12113 (2013).

27. C. Frantz et al., J. Cell Biol. 183, 865-879 (2008). 
28. S. Köhler, K. M. Schmoller, A. H. Crevenna, A. R. Bausch, Cell Rep. 2, 433-439 (2012).

29. C. Martin, S. F. Pedersen, A. Schwab, C. Stock, Am. J. Physiol. - Cell Physiol. 300, 490495 (2011).

30. H. X. Zhou, X. Pang, Chem. Rev. 118, 1691-1741 (2018).

31. G. M. Allen, A. Mogilner, J. A. Theriot, Curr. Biol. 23, 560-568 (2013).

32. M. Poo, Annu. Rev. Biophys. Bioeng. 10, 245-276 (1981).

33. M. Lange, J. Prassler, M. Ecke, A. Müller-Taubenberger, G. Gerisch, J. Cell Sci. 129, 3462-3472 (2016).

34. H. Zhan et al., Dev. Cell. 54, 608-623.e5 (2020).

10 35. L. E. Hind, W. J. B. Vincent, A. Huttenlocher, Dev. Cell. 38, 161-169 (2016).

36. S. Srinivasan et al., J. Cell Biol. 160, 375-385 (2003).

37. X. Sun, et al., 1 (2015), doi:10.1073/pnas.1502970112.

38. X. Sun, et al., Sci. Rep. 8, 1-9 (2018).

39. T. Kanungo et al., IEEE Trans. Pattern Anal. Mach. Intell. 24, 881-892 (2002).

40. R. Lee, et al., Mol. Biol. Cell, mbcE19110614 (2020).

Acknowledgments: We thank Q. Qing and M. Zhao for the discussion and B. Sharif for technical support. Funding: This work was funded by the Air Force Office of Scientific Research grant FA9550-16-1-0052. Author contributions: W.L, P.N.D, and J.T.F. conceived the project. Q.Y. did the electrotaxis experiment and data analysis. Y.M. did the Lattice LightSheet microscope experiment. M.J.H prepared the nano-topographic surfaces. Y.M., L.J.C., M.J.H., and A.L.B. participated in the interpretation of data analysis. Q.Y., P.N.D., J.T.F. and W.L. wrote the paper. Competing interests: The authors declare that they have no

\section{Supplementary Materials:}

Materials and Methods

Figures S1-S5

Movies S1-S8 

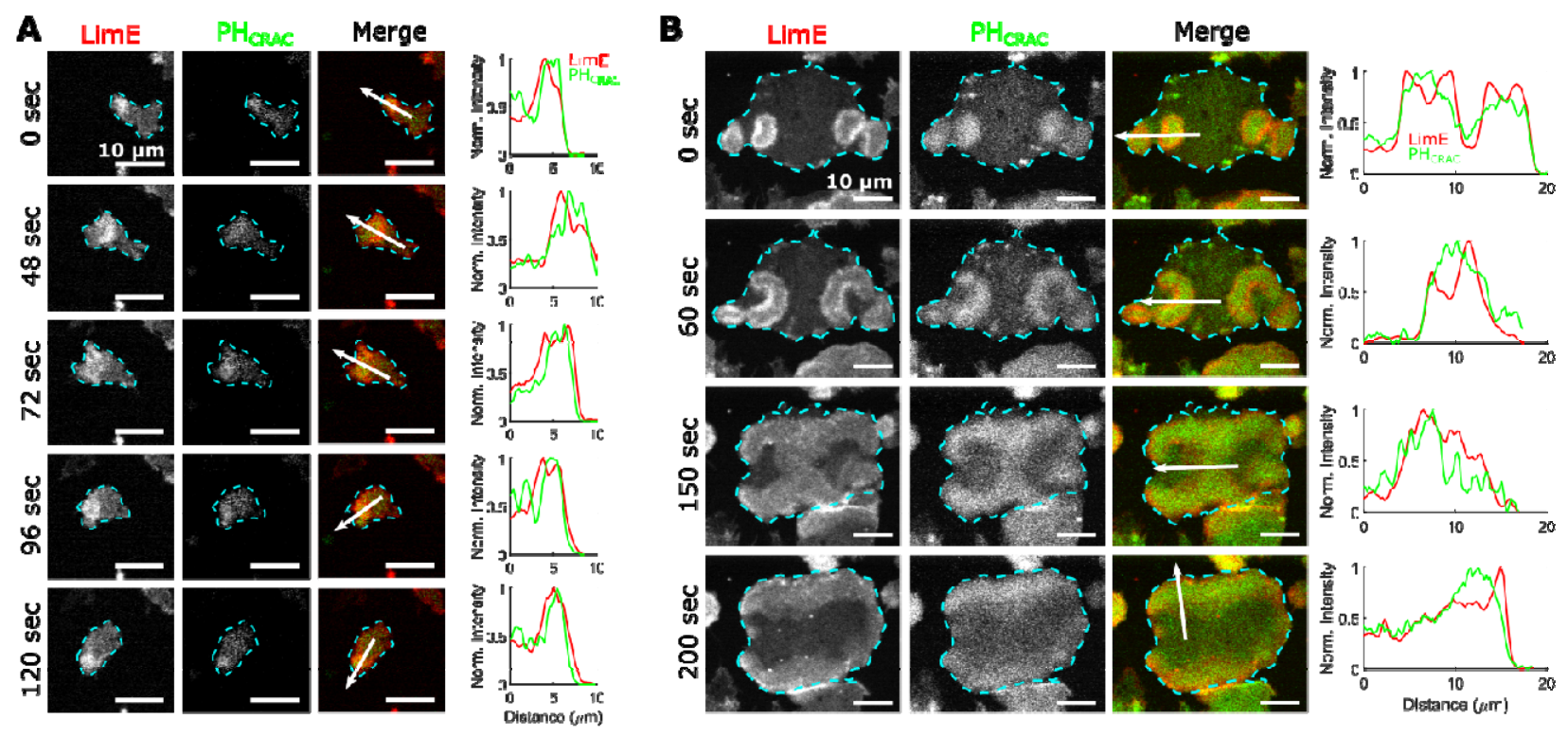

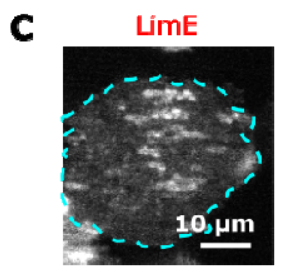

Merge
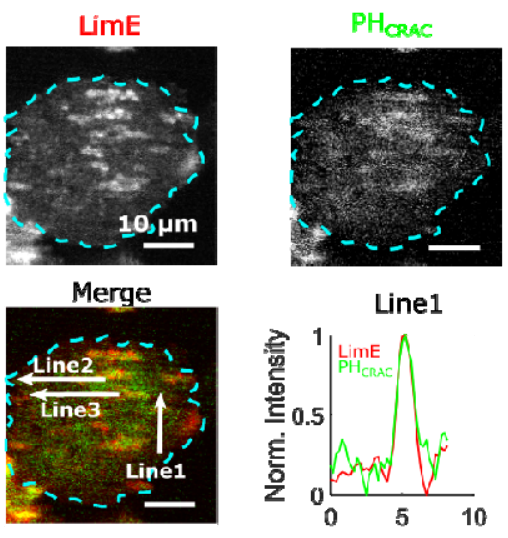

Line1

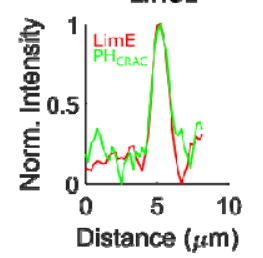

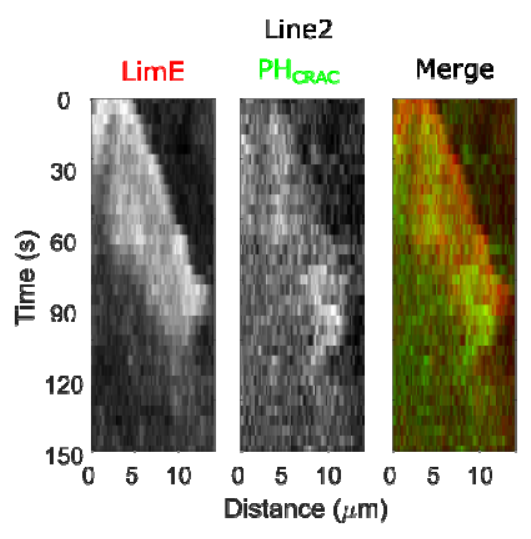
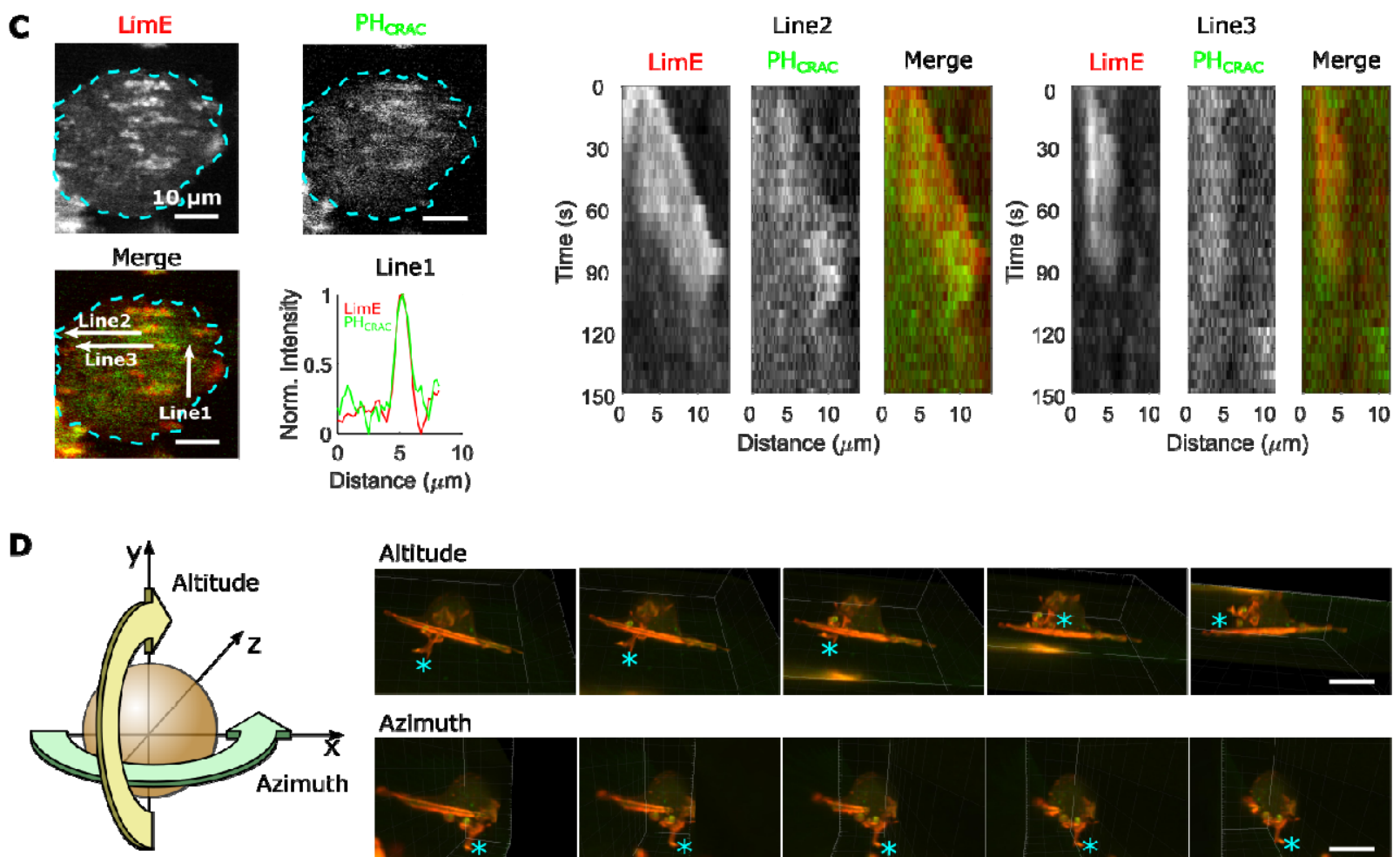

Altitude
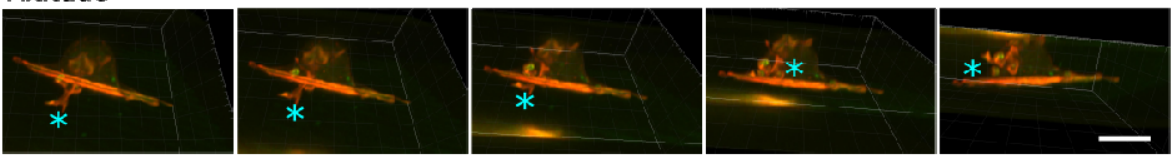

AzImuth

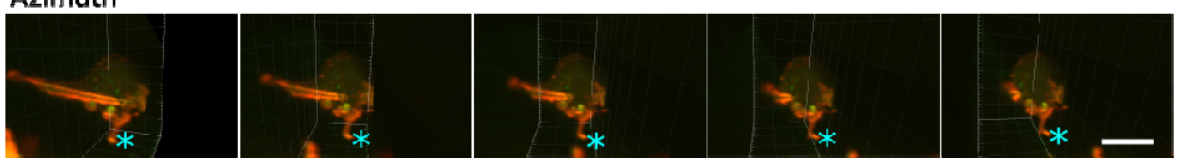

Fig. 1. STEN-CEN waves in single cells and giant cells. A. Snapshots of a differentiated, single $D$. discoideum cell expressing limE-RFP and PHcrac-GFP, with cell boundaries overlaid as blue dashed lines. The right column shows the normalized intensity of limE and PHcrac from the arrows in the merge images. B. Snapshots of an electrofused giant $D$. discoideum cell on a flat surface, with scanning profiles in the right column. C. A snapshot of an electrofused giant cell on the ridged surface. The left kymographs are from the line 2 and line 3 specified in the merged image. D. 3D reconstruction of a single $D$. discoideum cell on nanoridges, acquired using a Lattice LightSheet microscope. The reconstruction was rotated in two directions, as indicated in the schematic. The blue asterisks mark the location of one dorsal wave. The red channel represents limE-RFP and the green channel represents PHcrac-GFP. All scale bars are $10 \mu \mathrm{m}$. 

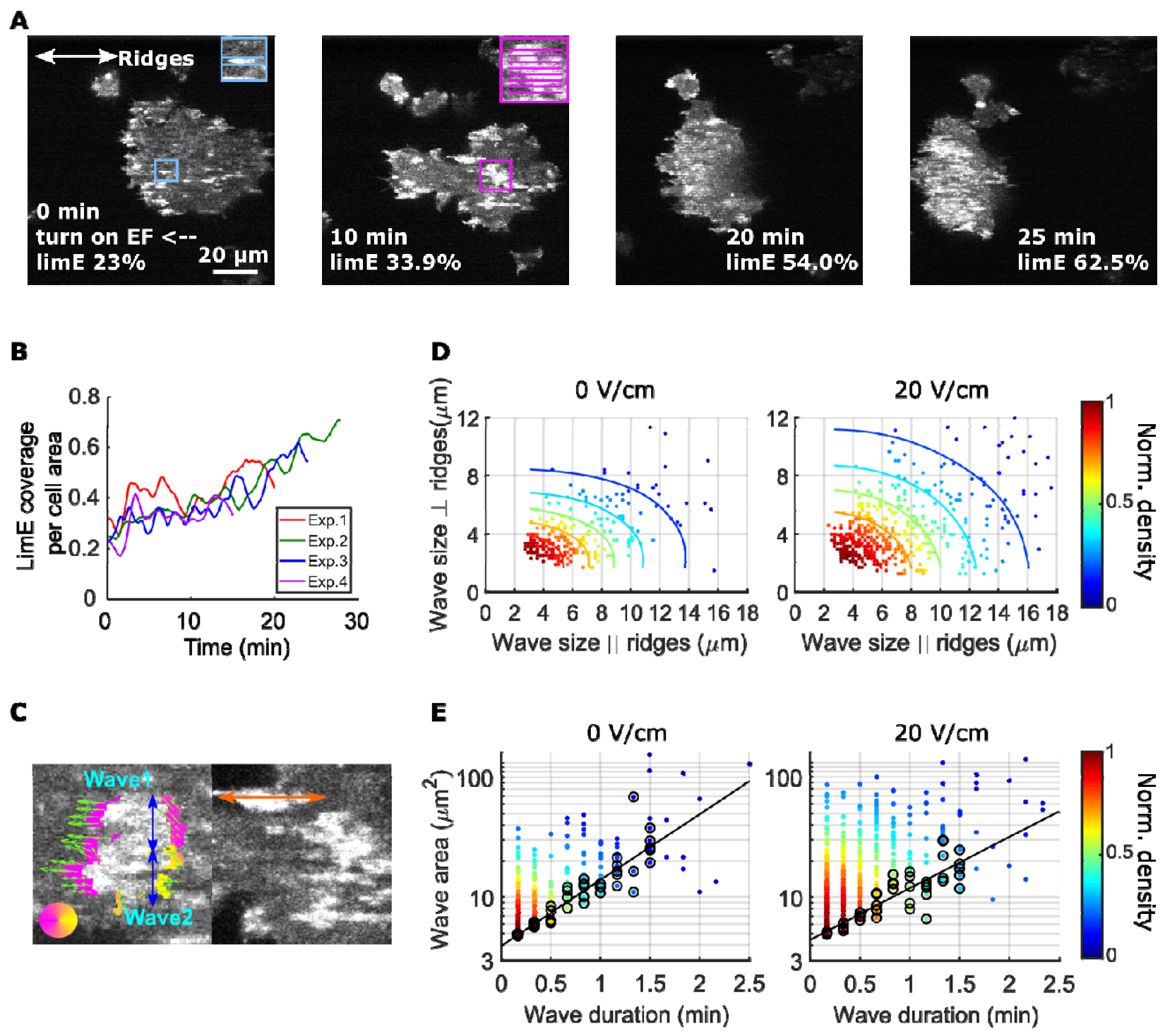

$\mathbf{F}$

$\mathbf{G}$
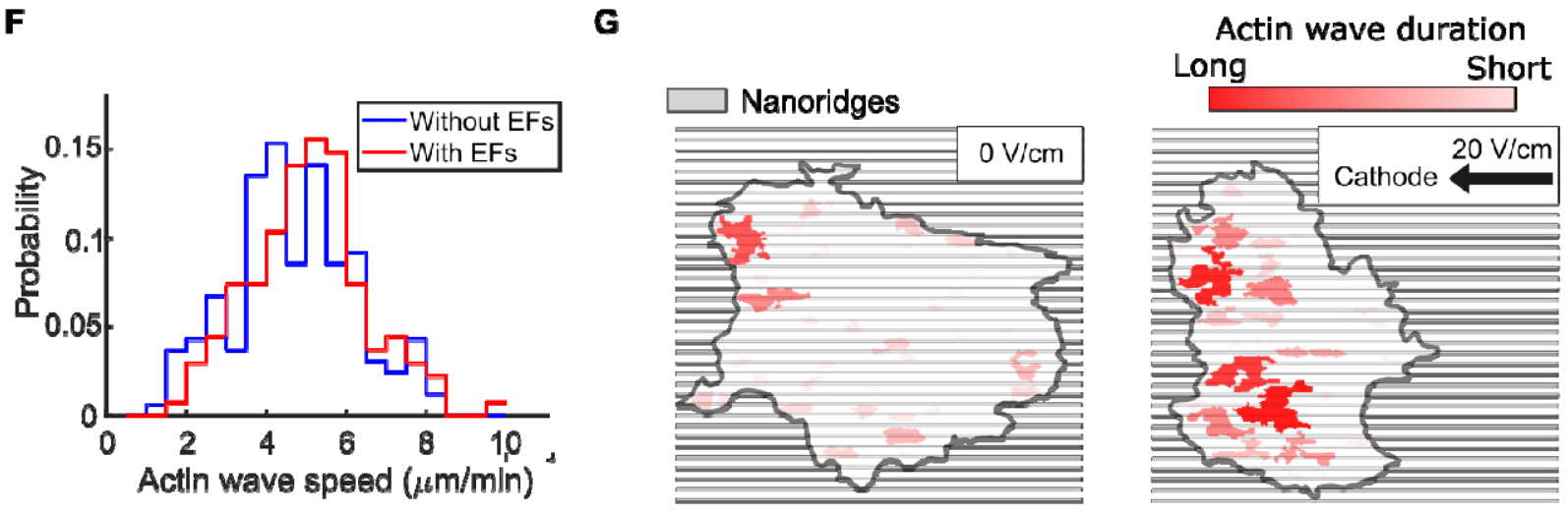

Fig. 2. EFs alter F-actin wave properties. A. Snapshots of limE images of a giant cell in an EF. The percentage of the cell area occupied by limE is indicated in each image. The EF was turned 
on at 0 min B. The temporal changes of limE-RFP occupancy per cell area in an EF that was introduced $0 \mathrm{~min}$. C. Division of groups of waves. The color represents the orientation of opticalflow vectors according to the color wheel. The left image is an example of a large structure composed of two independent substructures, where the vectors at the right edge are not moving in the same direction. The wave scales in the directions perpendicular to ridges (blue arrows) and parallel to ridges (orange arrow) were measured on the preprocessed waves. D. Density scatter plots of wave scales parallel to ridges vs. perpendicular to ridges. E. Density scatter plots of actin-wave dimension vs. actin-wave duration. For each wave duration, the 5 points with the smallest wave areas (black circles) were selected to fit the boundaries (black solid lines). F. Distributions of wave propagation speeds before (blue) and after (red) applying an EF. G. Schematic illustration of actin-wave activity in giant cells under without and with an EF. Actin waves with larger area and longer duration are generated in an EF. 
A

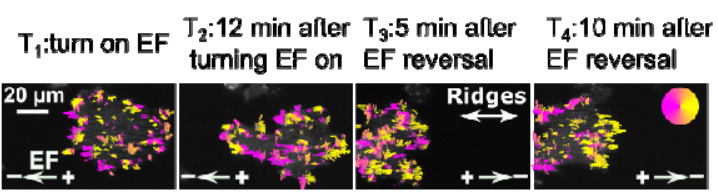

Proportion

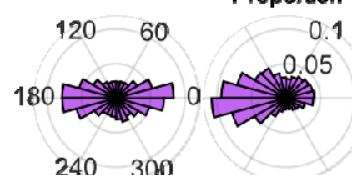

$240 \quad 300$

C

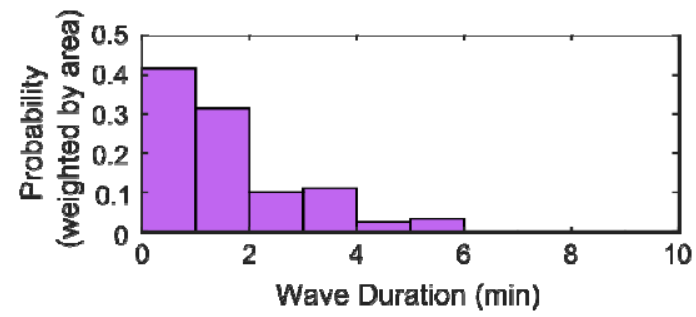

E

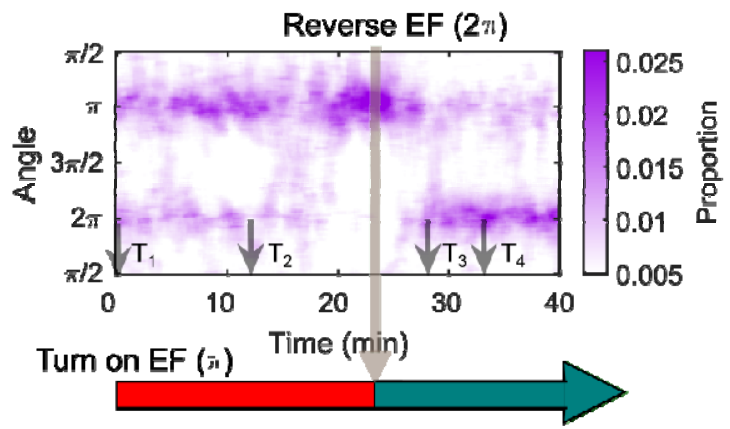

G

Directed migration in EFs

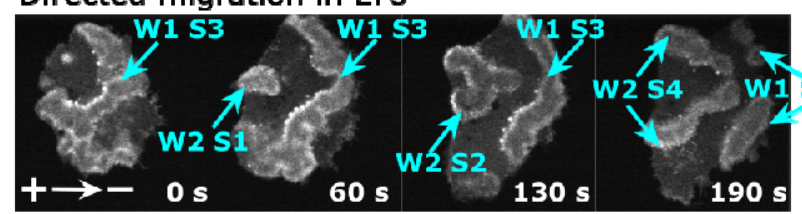

EF Reversal

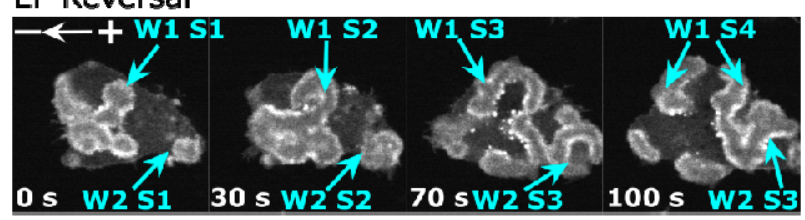

B
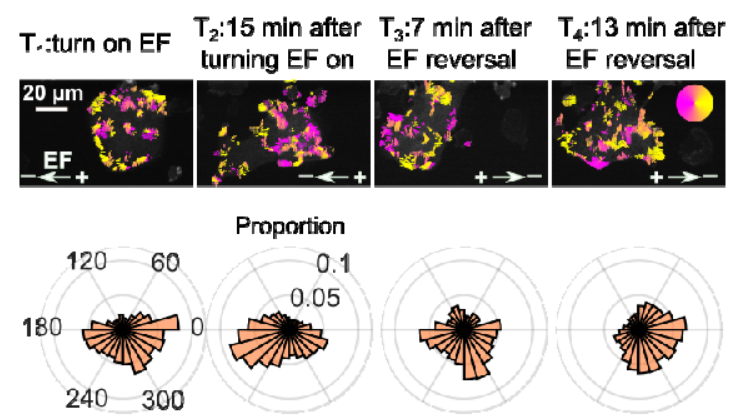

Proportion
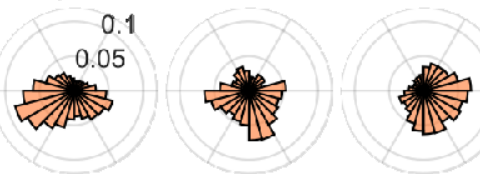

D

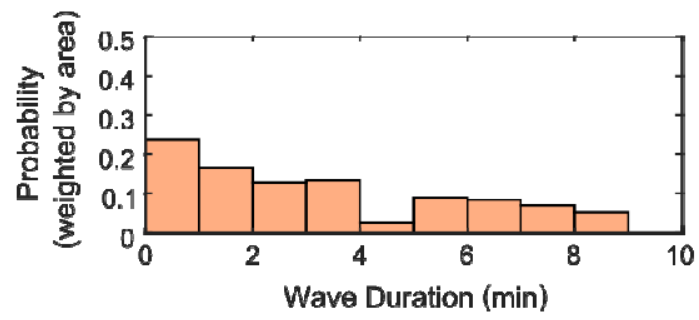

$\mathbf{F}$

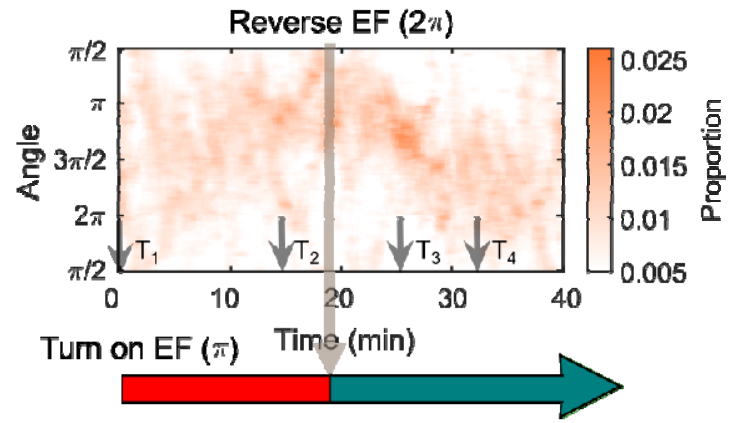

Actin wave $\rightarrow$ Wave propagation direction

Directed migration in EFs $+\longrightarrow-$
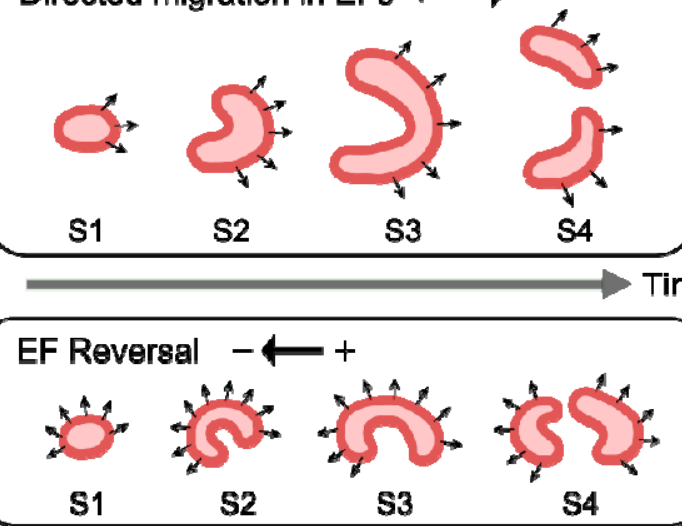

Fig. 3. Actin waves are guided by EFs. A, B Optical-flow analysis of actin-wave dynamics in giant cells on ridged and flat surfaces. The top row shows a time series of limE images for giant cells overlaid with optical-flow vectors, the color of which is coded according to the color wheel. The lower row shows the corresponding orientation displacements of optical-flow vectors. C, D Distributions of wave duration. The distribution was weighted by wave area, because the number of long-lasting large waves on flat surfaces is smaller than the number of short-lived small 
patches on ridged surfaces; correspondingly, the absolute counts of waves do not match the pixel-based, optical-flow analysis in A-B. E, F Kymographs of orientation displacements of optical-flow vectors. The $x$-axes of the kymographs represent time and the $y$-axes represent orientation. The colors represent the proportions. The EF was turned on at time $T_{1}$, and was reversed at the time denoted by the black arrow. G. LimE snapshots showing the patterns of actin wave expansion during steady directed migration in a constant EF (top) and after reversing the EF direction (bottom). The blue arrows point to certain stages of wave expansion. Abbreviation: W: Wave, S: Stage of wave expansion. H. Cartoon illustrating the patterns of actin-wave expansion during directed migration in EFs (top) and after EFs were reversed (bottom). 
A

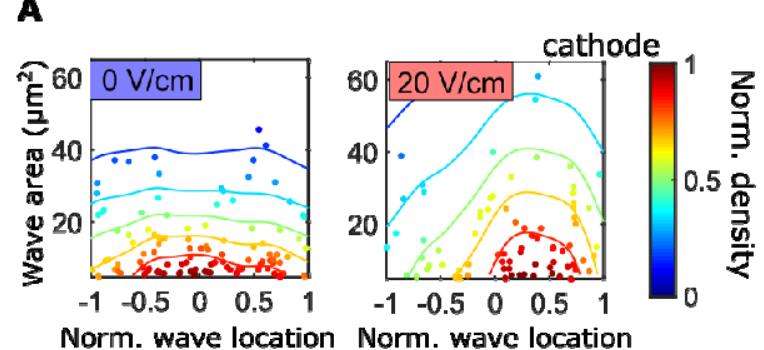

(by cell size)

(by cell size)

B

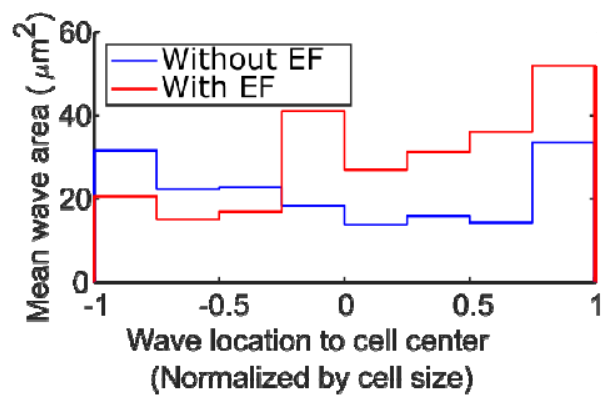

C

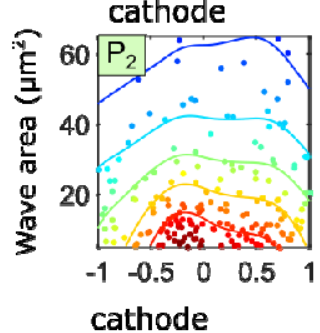

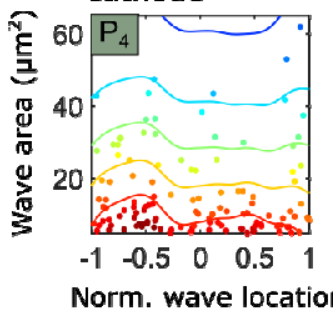

(by cell size)

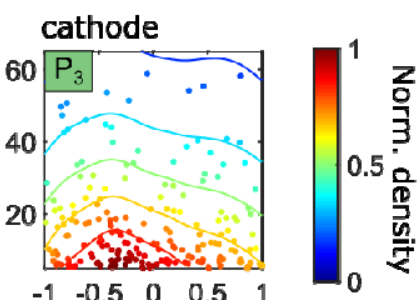

$\begin{array}{lllll}-1 & -0.5 & 0 & 0.5 & 1\end{array}$

cathode

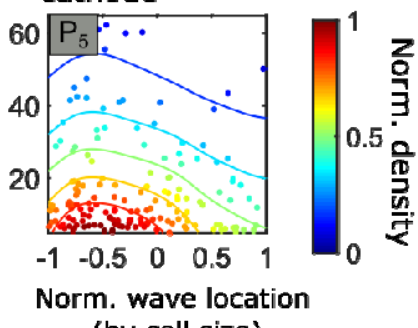

(by cell size)

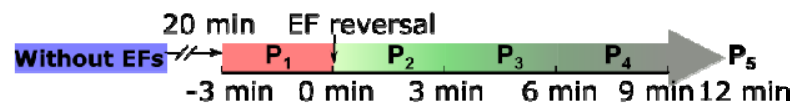

D

E

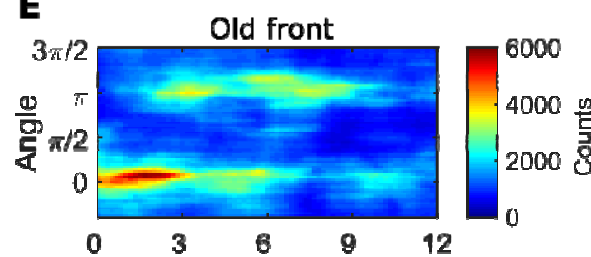

Reverse EF to the left

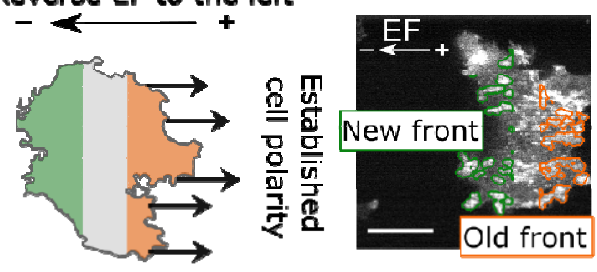

New front: Facing the new cathode

Old front: Facing the original cathode

G

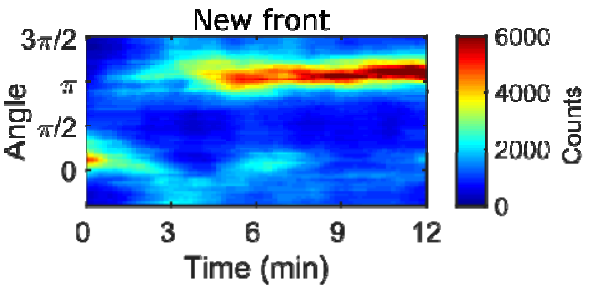

F

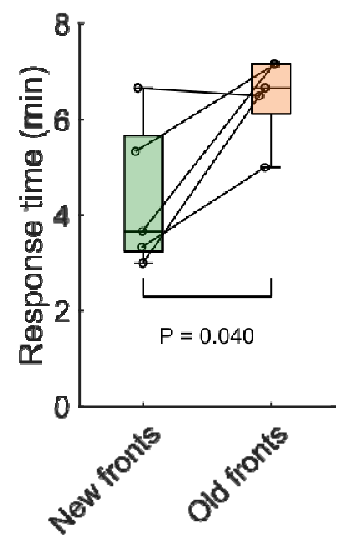

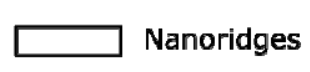

Nanoridges
STEN-CEN thresholds
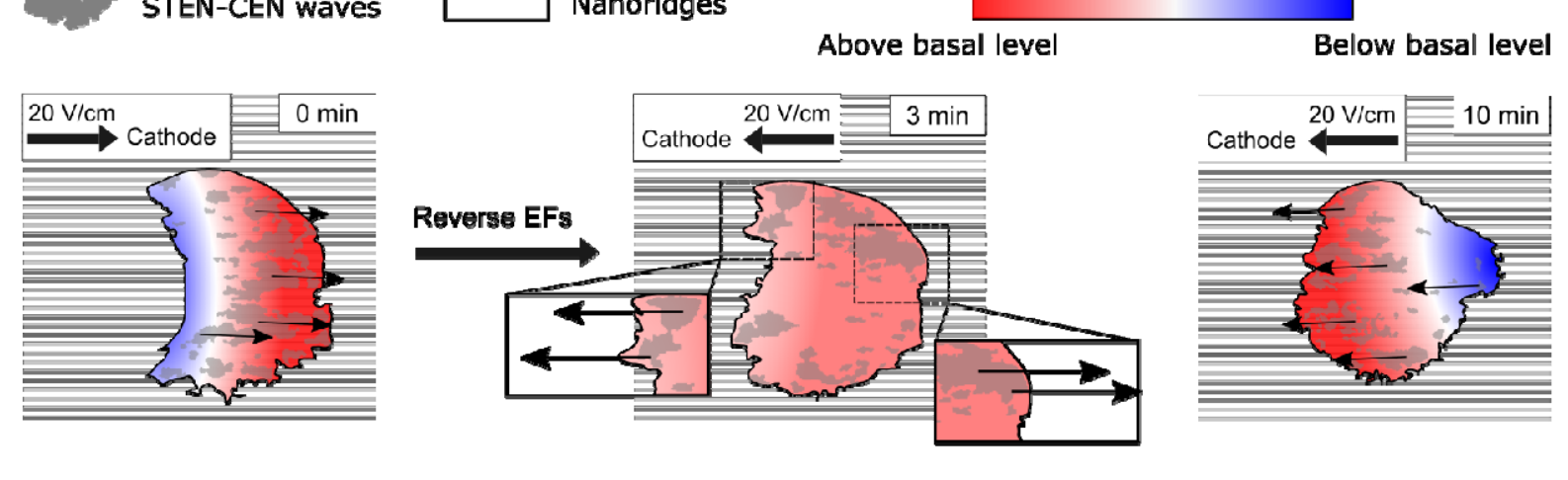

Fig. 4. Spatial inhomogeneity of the response to EFs. A. Density scatter plots of wave locations vs. wave areas. The left plot is for a period without an EF, and the right plot for the period in which cells exhibited steady directional migration. The $x$-axis is the location of each actin wave in reference to the cell center and the $y$-axis is the wave area. Color is coded 
according to the density of points. B. The basal region was binned into sub-regions at an interval of $10 \mu \mathrm{m}$. The average actin wave area in each bin is shown. C. Changes of spatial distribution of actin waves in response to EF reversal. The color of each plot is coded according to the timeline displayed at the bottom of C. $P_{2}-P_{5}$ : The EF was reversed and cells gradually developed polarization towards the new cathode. D. A schematic illustrating the old and new fronts of giant cells when the EF is reversed. E. Time stacks of orientation distributions of optical-flow vectors at old front and at new front. The EF was reversed from cathode at right (0) to cathode at left $(\pi)$ at $0 \mathrm{~min}$. F. Comparisons of response time between new fronts (green) and old fronts (orange) from multiple experiments $(N=5)$. G. Cartoon illustrating different time scales of local wave propagation and global rearrangement of STEN-CEN thresholds, in response to EF reversal. 